\title{
HIGH-RESOLUTION ANIMATION OF FACIAL DYNAMICS
}

\author{
N. Nadtoka, J.R. Tena, A. Hilton, J. Edge \\ Centre for Vision, Speech and Signal Processing, University of Surrey \\ $\{$ N.Nadtoka, J.Tena, A.Hilton\}@ surrey.ac.uk
}

\section{Keywords:}

\begin{abstract}
This paper presents a framework for performance-based animation and retargeting of high-resolution face models from motion capture. A novel method is introduced for learning a mapping between sparse 3D motion capture markers and dense high-resolution 3D scans of face shape and appearance. A high-resolution facial expression space is learnt from a set of 3D face scans as a person specific morphable model. Sparse $3 \mathrm{D}$ face points sampled at the motion capture marker positions are used to build a corresponding low-resolution expression space to represent the facial dynamics from motion capture. Radial basis function interpolation is used to automatically map the low-resolution motion capture of facial dynamics to the high-resolution facial expression space. This produces a high-resolution facial animation with the detailed shape and appearance of real facial dynamics. Retargeting is introduced to transfer facial expressions to a novel subject captured from a single photograph or 3D scan. The subject specific highresolution expression space is mapped to the novel subject based on anatomical differences in face shape. Results facial animation and retargeting demonstrate realistic animation of expressions from motion capture.
\end{abstract}

\section{Introduction}

Producing convincing 3D animated models of real facial dynamics for speech and expression is a difficult problem due to the sensitivity of human perception to the nuances of facial appearance and movement. In film production two approaches are used to achieve believable facial animation of real actors: highly skilled manual animation using key-frame face poses; and performance driven animation which maps the movement of an actors face captured using visual markers onto a 3D face model [11]. Performance-based animation can achieve highly realistic facial dynamics but requires mapping the movement of sparse marker points to the high-resolution model. Manipulation of detailed facial models to achieve realistic animation from motion capture is a highly skilled and time consuming manual process. In this paper we introduce a framework for the automated production of highly detailed facial animation of expressions from motion capture data, illustrated in Figure 1.

The proposed approach combines capture of a set of static 3D scans of facial expressions for a specific person, giving high spatial resolution of shape and appearance $(\approx 100 K$ points) but no temporal information, with marker-based motion capture sequences which give high-temporal resolution $(100-400 H z)$ but low spatial resolution $(15-100$ points $)$. Static 3D scans of facial expression have sufficient spatial resolution to capture fine surface detail together with texture mapped appearance. Conversely, motion capture of a sparse set of marker points captures the gross shape dynamics of facial deformation but does not have sufficient spatial resolution to capture fine surface details. The objective of the framework proposed in this paper is to achieve high-resolution animation of faces with the detail of static 3D scans together with the natural dynamics of motion capture. A methodology is presented to automatically map from a low-resolution space of facial expression dynamics to a high-resolution facial expression space. Corresponding low and high resolution expression spaces are learnt from the captured 3D face scans for a specific individual. This mapping allows the rendering of facial animations with high-resolution shape and appearance from a motion capture performance. Facial animation reproduces the learnt details of skin deformation learnt from the static scans whilst reproducing the natural dynamics of the subject's performance. This provides an automated procedure for high-resolution performance-based facial animation without the requirement for highly skilled manual instrumentation of a facial model.

The learnt model is person specific requiring a set of 3D scans of facial expressions together with the motion capture of the subject. To facilitate the animation of a different person from a single photograph or $3 \mathrm{D}$ scan we introduce a method of re-targeting the learnt model based on anatomical difference in facial shape. In the case of a single photograph as input an inter-person morphable model of facial shape and appearance [1] is used to reconstruct the novel subject. Retargeting the learnt model allows performance based animation of a novel subject from the motion capture of the original person with detailed skin deformation derived from the person specific model. Results are demonstrated for facial animation of celebrities derived from a single photograph. Contributions of the proposed framework include:

- A method for learning a mapping between sparse 3D motion capture data of facial dynamics and high-resolution static 3D face scans.

- Automatic high-resolution animation of facial dynamics from motion capture with the detailed wrinkles and facial appearance captured in static 3D scans.

- Retargeting of captured facial dynamics to novel subjects 


\section{Subject specific animation model $\begin{gathered}\text { Inter-subject } \\ \text { animation model }\end{gathered}$}

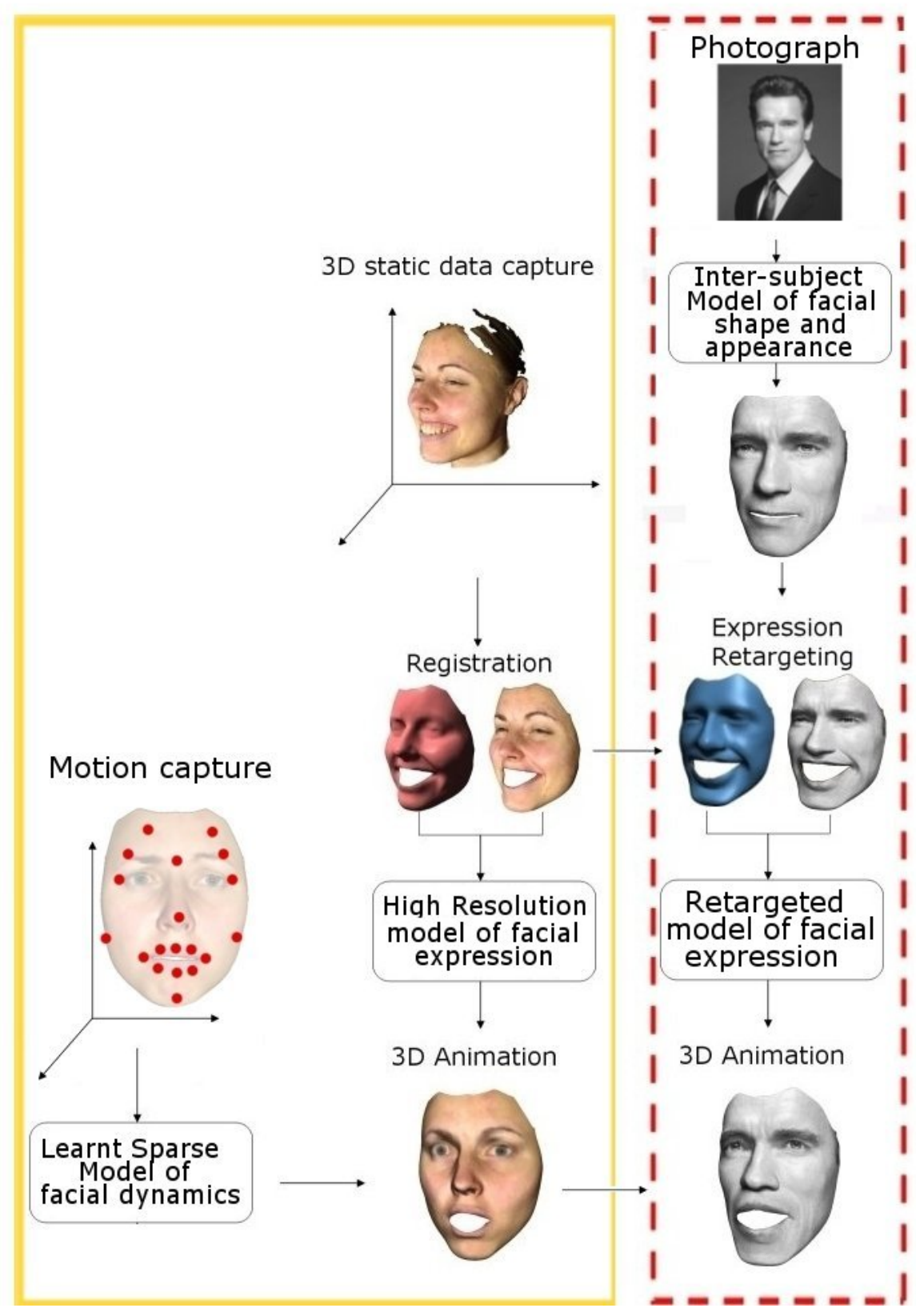

Figure 1: An overview of presented approach.

in order to animate high resolution models from a single photograph.

Our method overcomes limitation of previous performance based animation techniques requiring highly skilled and time-consuming manipulation for animation of facial models from motion capture. Currently the approach is limited to animation of facial expressions, future work will address the high-resolution animation of speech and emotion with natural facial dynamics.

\section{Background}

There have been a wide range of techniques for animating human facial models since Parke's [10] seminal work on parametric models. These methods can be categorized by how they attempt to represent and control the face (e.g. geometric deformation vs. physical models of the face) and how these models are manipulated over time to produce animation (e.g. morph-targets vs. data-driven approaches).

Geometric free-form deformation techniques directly 
manipulate the surface of a model using intermediary controls such as points [8], splines [14], and surface patches [15]. As these controls are manipulated the deformation is transferred to the target facial surface. In contrast, physicallybased techniques attempt to mimic the structure and function of the human face. Most commonly the elastic nature of facial skin is modelled using mass-spring systems [6] or finite-element models [5]. Unfortunately, physical models of the face tend to be very computationally expensive, reducing their applicability to real-time animation systems.

Another form of facial modelling relies upon building statistical representations of the face from multiple samples of different expressions. A common technique used for this is principal component analysis (PCA) which can create an orthogonal basis for facial expression based upon the statistics of the covariance matrix [1]. PCA is beneficial because it can also be used for compression and denoising of the facial model by culling components corresponding to low variation in the original samples. Other techniques for dimensionality reduction have been proposed including independent component analysis (ICA) [7] and non-linear methods such as Isomap [4].

Animation techniques define how the controls of a facial model are varied over time to reproduce correct dynamics. Traditionally, morph targets are used to produce animation, where each expression in the sequence is a finite combination of pre-modelled expressions. However, morph-based techniques do not conform well to how real facial expressions change over time. Motion capture allows points on the face to be accurately tracked over time, and these points can be used to manipulate a facial model directly [18]. Whilst most animation produced is subject-specific (i.e. fixed to the identity of one person) it can be beneficial to define techniques to transfer animation between models of different individuals. This retargeting of facial animation is typically performed by defining a mapping between the two individual models and using this mapping to transfer motion vectors embedded at the vertices [9] or control points [13].

\section{Framework for high-resolution facial animation from sparse capture}

\subsection{System overview}

The work presented here can be subdivided into two clear parts (as shown in the figure 1):

1. Subject specific animation model.

- Learnt sparse model of facial dynamics

- Model of high resolution facial shape and appearance.

2. Retargeting animation to a novel subject.

- Inter-subject morphable model of shape and appearance
- Learnt intra-person model of facial expressions for 3D shape and appearance

Person-specific model of facial dynamics A high-resolution dataset for a single subject consisting of static 3D scans of a chosen set of expressions (happiness, sadness, anger, disgust, surprise, fear and neutral) is collected for several degrees of intensity in each expression. The following registration step brings all the input meshes in one-to-one correspondence with a generic facial model mesh. The registered texture maps are estimated given a mapping from the generic mesh to the texture coordinates. The statistics of 3D facial surface variations are learned based on the morphable model of shape and texture. Spatially low resolution (19 markers) 3D motion capture recorded for the same subject is used here to learn the dynamics of facial expressions. The combination of both statistical models (3D scans and 3D motion capture) gives a framework for production of high resolution 3D animation of faces from motion capture.

Retargeting of animation to novel subjects The approach discussed above is extended to retargeting of captured facial dynamics to different subjects. The 3D shape and appearance of a new subject is estimated from a single photograph with an inter-person morphable model framework [1]. Similarly to the first part of method, the resulting 3D expression scans are used to learn the statistics of facial surface changes. The mapping between the facial dynamics of the first performer and the high resolution model of 3D scans of facial expressions for the second subject is learned, which allows automatic retargeting of a facial dynamics between the subjects.

\subsection{Parameterization}

This work combines two different types of 3D data:

- 3D static scans, which offer high geometric fidelity of the mesh, but low temporal resolution.

- Motion capture (mocap) data, which can be recorded at a high framerate, but only provides spatially low resolution data.

The presented system consists of two morphable models: 1) a model of the low resolution mocap data; 2) a model of the high resolution scans.

To enable combination of these two data sources we register both with a common generic model. Inter-person statistics are then learnt. The mapping between these spaces is defined automatically from a number of known correspondences within both model spaces.

\subsubsection{High-resolution expression model space}

45 high-resolution static 3D scans of 7 basic facial expressions (with several degrees of intensity) were recorded with 

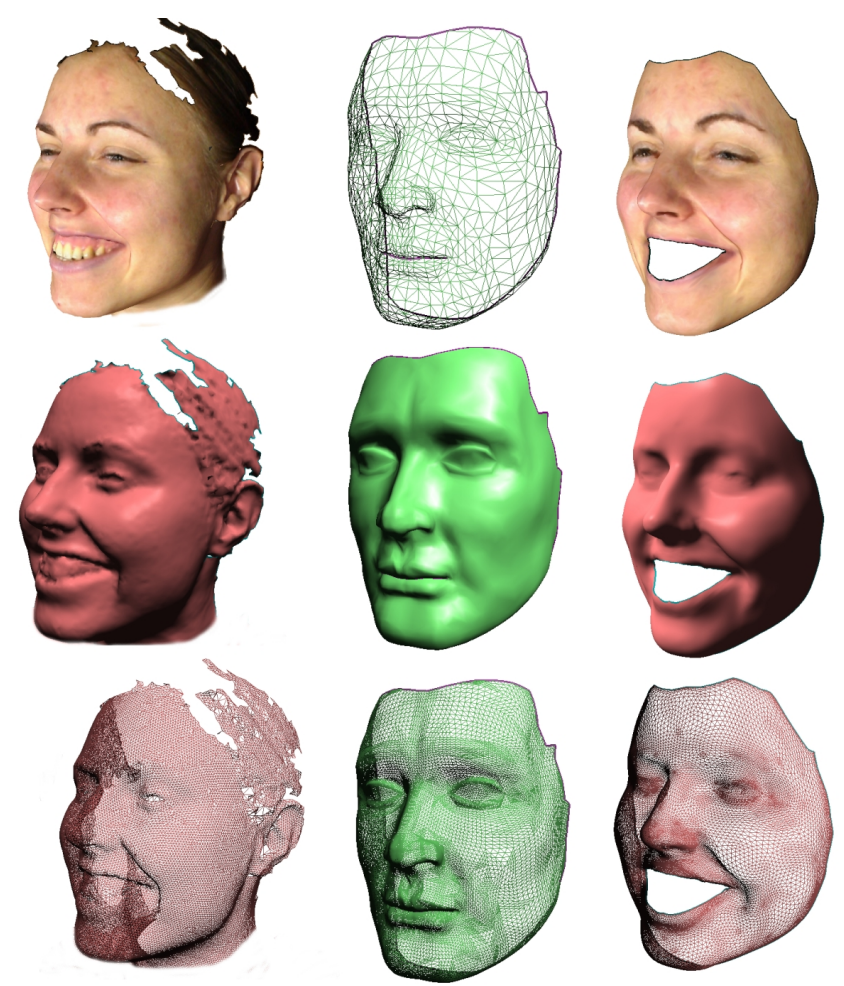

Figure 2: From left to right: input $3 D$ scan(with texture, shaded and mesh representation), generic mesh (low resolution, shaded and twice subdivided), output registered $3 D$ face (with texture, shaded and mesh)

commercial 3D scanner (3dMDFace ${ }^{T M}$ System). The facial meshes vary in the number of vertices and mesh structure, therefore a registration procedure (see fig. 2) is needed in order to assure one-to-one correspondence.

Deformable face model registration A set of landmark points is labelled on generic mesh and every $3 \mathrm{D}$ expression scan. These points are chosen to mark distinctive facial features (e.g., tip of the nose). The landmarks points are set at the same locations as 3D motion capture markers during mocap data collection step. We refer to the landmark points as $D_{i}^{s u b}$ for i-th face scan (see fig. 3). Selecting the points for registration assures that these mesh coordinates undergo minimal changes (less than $0.1 \mathrm{~mm}$ of magnitude) during the registration process. We use the registration method presented in [17] which can be separated into three main stages: global mapping; local matching; energy minimization.

During the global mapping stage, the sets of landmarks of the generic mesh and a 3D scan are aligned using the thin plate spline interpolation technique [3], that smoothly deforms the generic mesh in order to minimize the bending energy. The second stage comprises a search for the most similar point on the $3 \mathrm{D}$ scan for every vertex on the generic mesh within a given search radius of $10 \mathrm{~mm}$. The similarity measure between two vertices is defined by a weighted sum of the distance between

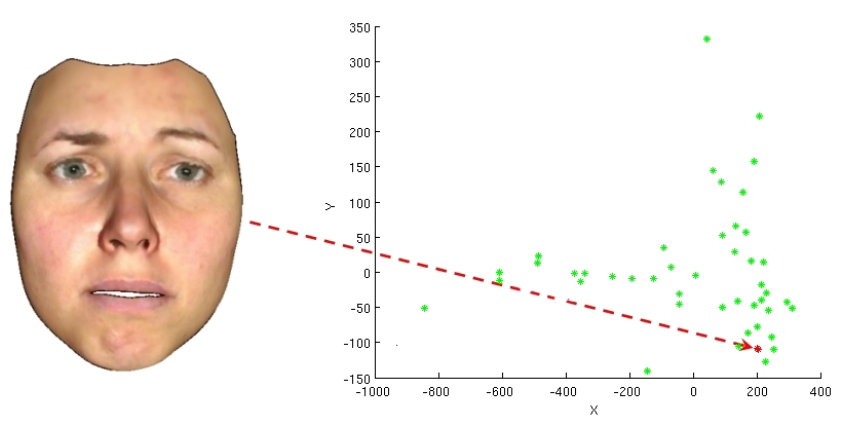

Figure 4: First 2 principal components of $3 D$ shape morphable model. The green stars mark the locations of original 3D scans.

them, the angle between their normals, and the difference between their curvatures. The final set of matches is used to guide the energy minimization process that deforms the generic mesh to the 3D scan. More details can be found in [17]. The registration process is illustrated in figure 2 . Texture maps are obtained by warping the original texture image to the generic mesh texture coordinates. A resolution of 6600 vertices was found to be sufficient to preserve the facial surface detail close to the one which was present in the $3 \mathrm{D}$ scanner data.

High resolution model of facial 3D shape and appearance Given the registration of all 3D scans with a common 3D model $D_{i}$ the morphable model is constructed. The learnt model used here is a variation of work of Blanz and Vetter [1]. PCA is used here to learn the statistics of facial surface and texture changes with expression from 3D scans. This allows us to extract the common modes of variation within the input static data scans. A 3D shape of a face can be presented as a vector consisting of $x, y, z$ triplets $D_{i}^{s}=\left(x_{1}, y_{1}, z_{1}, x_{2}, y_{2}, z_{2}, \ldots, x_{m}, y_{m}, z_{m}\right)^{T}, i=\in 1,45$. Similarly a single texture can be presented as a vector of R,G,B triplets $D_{i}^{t}=\left(R_{1}, G_{1}, B_{1}, R_{2}, G_{2}, B_{2}, \ldots, R_{n}, G_{n}, B_{n}\right)^{T}$, $i=\in 1,45$. After performing PCA [12] each shape $D_{i}^{s}$ and texture $D_{i}^{t}$ can be represented within the model space as vectors of parameters:

$$
\begin{aligned}
\hat{D}_{i}^{s} & =\left(\alpha_{1}, \alpha_{2}, \ldots, \alpha_{M}\right) \\
\hat{D}_{i}^{t} & =\left(\beta_{1}, \beta_{2}, \ldots, \beta_{N}\right) \\
D_{i}^{s} & =\overline{D^{s}}+\sum_{j=1}^{M} \alpha_{i} \cdot d_{i}^{s} \\
D_{i}^{t} & =\overline{D^{t}}+\sum_{j=1}^{N} \beta_{i} \cdot d_{i}^{t}
\end{aligned}
$$

The number of principal components is then reduced to $m=$ 41 for shape and to $n=43$ for texture which preserves the accuracy level to $99 \%$ of the observed variance. Figure 4 shows the first two principal components of morphable model 

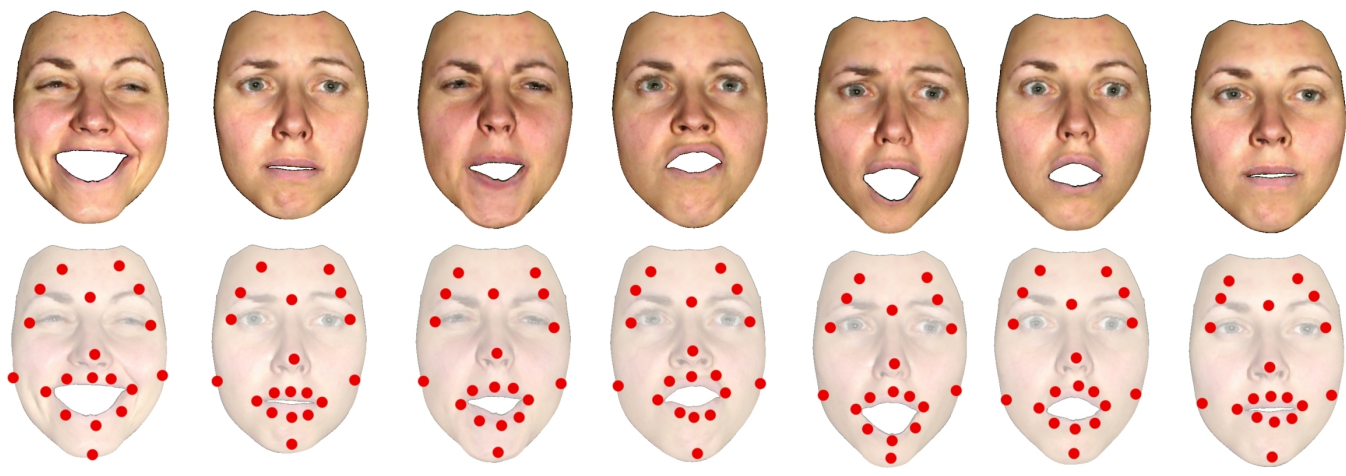

Figure 3: 3D expression scans are subsampled at the labelled locations which correspond to the positions of motion capture markers.

space. This model can be used to produce novel expressive face animations via key-frame interpolation. The parameters of this model are derived to produce a high resolution version of the face matching dynamics of an input mocap sequence

\subsubsection{Low resolution model of facial dynamics}

The motion data was captured using an infra-red optical motion capture system with active markers (Coda). 19 markers were placed on the face of performer. 3D markers positions were captured at a rate of 100 frames per second. The system delivers raw 3D data. The data is post processed to compensate for the noise and errors. To allow further statistical analysis, data is rigidly aligned to the same coordinate system. The recorded motion capture data used in this paper consists of a 30 seconds sequence and includes the transitions between selected expression states: happy, surprise, angry, neutral, sad, disgust and fear.

Sparse Model The PCA model described in the previous section is used to animate the face from captured motion data. We automatically select a set of key-frames $S^{s u b}$, from motion capture frames $S$ which have the minimal distance to the subset of 3D facial meshes $D^{s u b}$. The similarity measure is the sum of square differences. 3D motion capture key-frames $S_{i}^{s u b}, i=\epsilon$ 1,45 were rearranged into a vector form. Each vector is a $3 \mathrm{D}$ motion capture frame stored in terms of $x, y, z$ coordinates of all vertices $k, k \in 1,19$

$$
S_{i}^{s u b}=\left(x_{1}, y_{1}, z_{1}, x_{2}, y_{2}, z_{2}, \ldots, x_{k}, y_{k}, z_{k}\right)^{T}, i=\in 1,45
$$

Finally, we perform PCA to define a parametric space for the facial dynamics. The number of principal components is then reduced to $m=12$ to preserve $99 \%$ variance of original data. Each motion capture frame can be now represented as a linear combination of orthogonal eigenvectors:

$$
S_{i}^{\text {mocap }}=\bar{S}+\sum_{j=1}^{m} \gamma_{i} \cdot s_{i}
$$

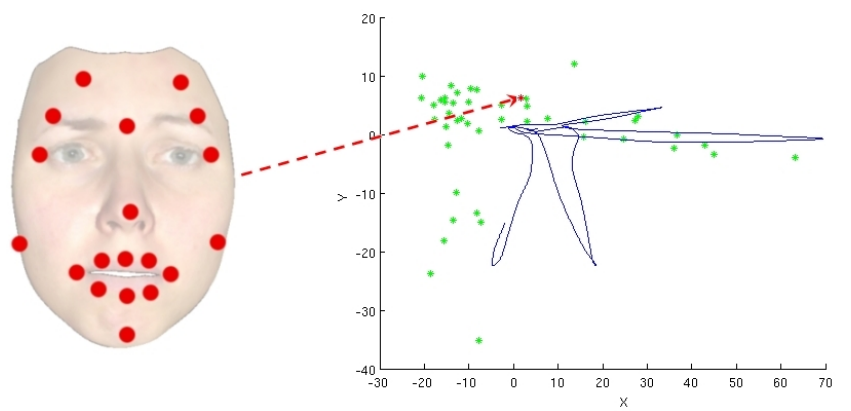

Figure 5: Motion capture representation within the model space plotted across two first principal components. Blue curve is the motion capture frames projected into the model space. Projections of subset of 3D facial meshes $\hat{D}_{i}^{s}, i=\epsilon$ 1,45 onto the model are marked with green stars.

Projection of motion capture frames onto the orthogonal basis of morphable model space results in each frame corresponding to a parameter vector within this space $\hat{S}_{i}^{\text {mocap }}=\left(\gamma_{1}, \gamma_{2}, \ldots, \gamma_{m}\right)$. Using the same orthogonal basis the subset of 3D facial meshes $D_{i}^{s u b}, i=\in 1,45$ is then also projected onto the model space. Figure 5 shows the first two principal components of the model space. The blue curve corresponds to the motion capture frames within the model space. The subset of $3 \mathrm{D}$ facial meshes $\hat{D}_{i}^{s u b}$ is marked with green stars.

\subsection{Low to High resolution Mapping}

\subsubsection{Correspondence}

There is a direct correspondence between the subset of 3D mesh data $D_{i}^{s u b}$ and full 3D mesh $D_{i}^{s}$. This results into the direct relation between their projections $\hat{D}_{i}^{s u b}$ and $\hat{D}_{i}^{s}$ into the sparse and dense model spaces:

- a parameter vector $\hat{D}_{i}^{s u b}=\left(\gamma_{1}, \gamma_{2}, \ldots \gamma_{m}\right)$ - a projection of $D_{i}^{s u b}$ into the sparse model space, that has been built based on the key-frames of motion capture. 


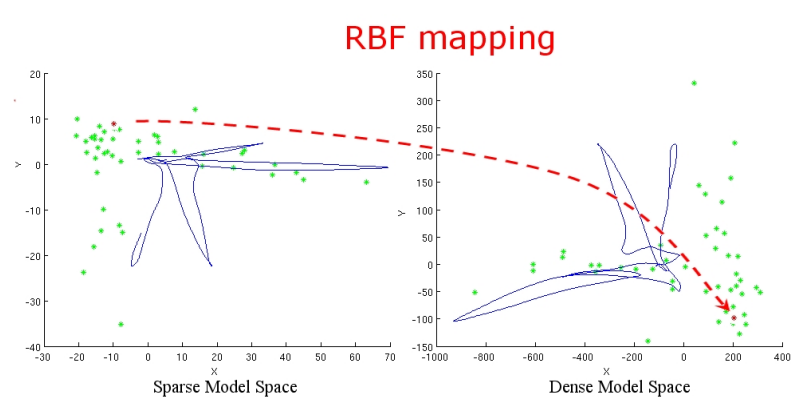

Figure 6: A mapping from sparse to dense model space.

- a parameter vector $\hat{D}_{i}^{s}=\left(\alpha_{1}, \alpha_{2}, \ldots, \alpha_{n}\right)$ - a projection of $D_{i}^{s}$ into the dense model space that has been constructed from the full 3D meshes.

The parameter vectors $\hat{D}_{i}^{s u b}$ and $\hat{D}_{i}^{s}$ are then rearranged into the matrix form: $\hat{D}^{s u b}$ and $\hat{D}^{s}$ correspondingly.

\subsubsection{RBF-mapping}

Based on the known correspondences between $\hat{D}^{s u b}$ and $\hat{D}^{s}$ we define an radial basis functions(RFB)-based mapping. In the generic case radial basis functions have the following form:

$$
\begin{gathered}
y(x)=\sum_{i=1}^{N} \omega \cdot \phi\left(\left\|x-c_{i}\right\|\right) \\
\phi(r)=r^{2} \cdot \log (r)
\end{gathered}
$$

Here the approximated function $y(x)$ is represented as a sum of $N$ radial basis functions, each associated with a different center $c_{i}$, and weighted by an appropriate coefficient $w_{i}$. In this work the thin plate spline is chosen as the basis function (see eq. 3.3.2), although, a similar results can be obtained with other choices of basis.

Based on the constructed mapping every parameter vector $\hat{S}_{i}^{\text {mocap }}$ that results from projection of motion capture data inside the sparse model space is projected onto the $\hat{D}_{i}^{\text {mocap }}$ parameter vector within the dense model space. A novel 3D face can be generated as a weighted sum of eigenfaces for each parameter vector. This means that a high resolution 3D mesh is associated with every motion capture frame. Moreover, RBFs have useful properties to preserve the relative distances between data samples during the mapping process, which preserves the realism of each generated animation sequence. As can be seen in figure 6 the trajectory of motion capture within the sparse model space has similar characteristics to its projection into dense model space. The motion capture trajectory is mapped to the texture morphable model in a similar fashion.

\subsection{Retargeting animation to a single 3D model or photograph}

It is useful to be able to cross-map animation recorded with motion capture data of one performer to animate a 3D face model (a single 3D scan or a number of scans) of another subject. In a generic case a single photograph of a second subject can be used to estimate a 3D scan of a second person. After the 3D scan is reconstructed the work follows the stages which are selected in the fig. 1 with the red rectangle: expression transfer, modelling and animation.
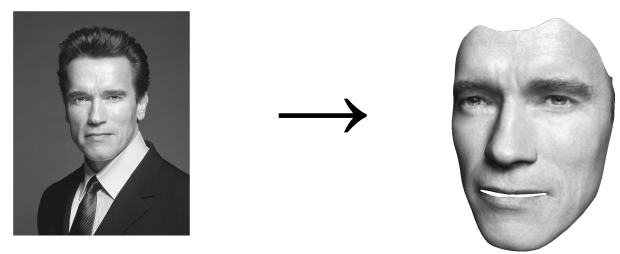

(a)
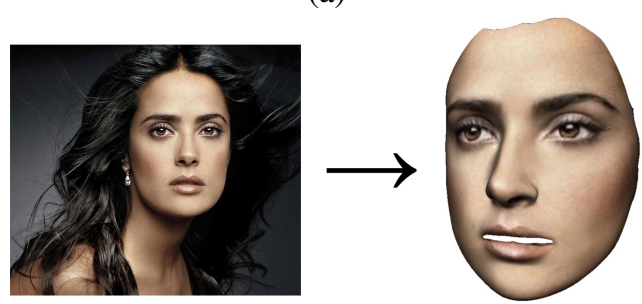

(b)

Figure 7: A single photograph is used to estimate a corresponding $3 D$ shape and texture information based on morphable model of identity.

To extract full 3D models from 2D photographs we used a morphable model based on the morphable model methodology described earlier in this paper. For more information on the methodology for constructing a morphable model the reader may refer to [2,1]. Our data base consists of 70 human faces of males and females of different age groups and races, and was collected using a $3 d M D F a c e^{T M}$ System. Since the database for building the morphable model was also registered using the method in [16]; any 3D reconstruction obtained by fitting the model to a 2D photograph can be readily used in the expression transfer process described in section 3.4.1. Fig. 7 (a) and (b) show the examples of $3 \mathrm{D}$ estimation results.

\subsubsection{Expression Transfer}

Expression transfer is done by measuring the vertex displacement that occurs between two different expressive states of a 3D face and then mapping the displacement to a different 3D face for which one of the two expressive states is known. In our work, we always use a neutral (expressionless) state as a reference from which we measure the vertex displacement to different expressions.

To transfer an expression from face $A$ to face $B$ we use a 

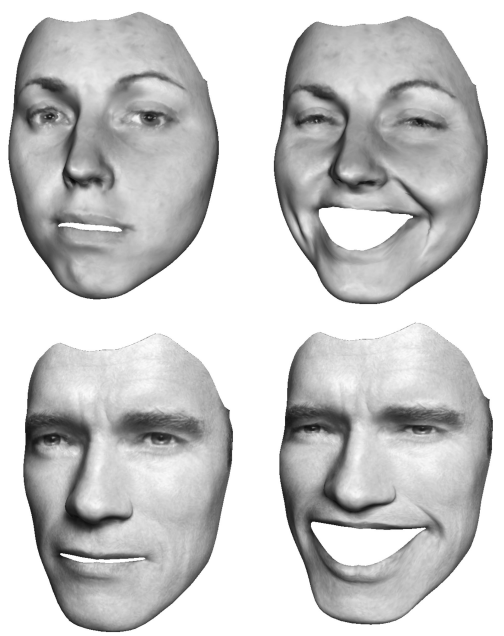

Figure 8: A scheme of expression transfer.
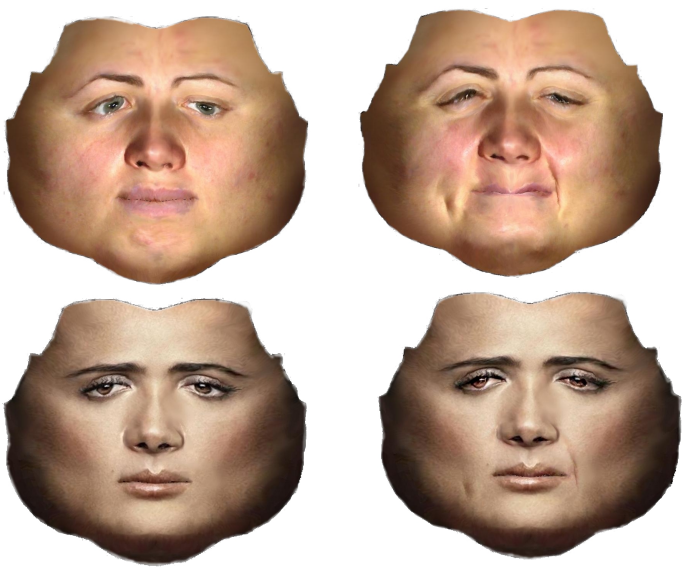

(a)
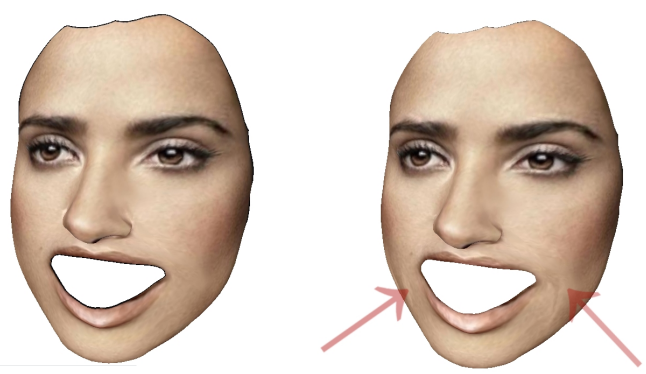

(b)

Figure 9: The masked texture differences between expression scan and neutral expressions are used to add the wrinkle detail.

neutral 3D sample of each ( $A N$ and $B N$ respectively) and a 3D sample of $A$ bearing the desired expression ( $A E$ ). A subset of 8 vertices is identified on the three samples: $A N, B N$ and $A E$. The subsets from $B N$ and $A E$ are aligned to the subset of $A N$. The resulting transformations are applied to $B N$ and
$A E$ respectively bringing all three samples to alignment. To estimate a sample of $B(B E)$ bearing the same expression as $A E$, a displacement vector $\overrightarrow{v_{i}}$ for each vertex $i$ is calculated by subtracting the $i^{t h}$ vertex of $A N$ from the $i^{t h}$ vertex of $B N$. $B E$ is then found by adding $\overrightarrow{v_{i}}$ to the $i^{t h}$ vertex of $B N$. Note that $\overrightarrow{v_{i}}$ can be weighted by a constant $k$ to emphasize $(k>1)$ or deemphasize $(0<k<1)$ the transferred expression. Since the anatomy of $A$ and $B$ may vary considerably the addition of the displacement vectors to $B N$ can cause structural distortion of the polygons in $B E$, and some times over-folding. To avoid this effect, local and global mean filtering is applied to $B E$. The local filter acts only on those polygons which are found to over-fold by replacing each vertex by the mean value of its connected second order neighbourhood. A Polygon is identified as over-folded when the signs of the $x, y$, and $z$ components of its normal in $B N$ are inverted in $B E$. The global filter replaces the value of each vertex by the mean value of its connected neighbours smoothing the mesh structure of $B E$. Figure 8 shows the result of transferring a smile from a $3 \mathrm{D}$ scan of a female to that of a male.

\subsubsection{Adding the Details}

The low level texture details such as wrinkles add significant realism to the animation. A procedure similar to the expression transfer of the shape deformations is applied to add the expression details in texture. The RGB textures may contain some lighting variations as well as expression specific color changes(wrinkles and skin folds), therefore a special care has to be taken while cross-mapping texture differences from actor $A$ to another subject $B$. A mask is used to select the regions of interest in the texture map specific to the wrinkles such as mouth furrows and the region around the eyes. To avoid the colour changes that may be specific to the skin colour of performer the texture differences are then converted to gray-scale. The resulting greyscale image is then added to RGB texture channels of the subject $B$. Figure 9 shows the results of adding the texture detail for the happy expression.

\subsubsection{Animation}

A straightforward way to cross-map the animation is to independently transfer the expressions from every frame of the dense 3D animation sequence. This approach gives plausible results; however, one drawback of it is that one needs to store every frame of the animation sequence. This can be solved by applying a parameterization scheme of the data which allows a compact representation of animation within the model space. Given that there is a one-to-one correspondence between expressions of person A and transferred expressions of person $\mathrm{B}$, our technique is extended to map between the motion capture trajectory within the sparse model space of person A to the person specific morphable model of expressions built for person B. A final animation of high resolution 3D scans of person $\mathrm{B}$ is produced by reconstructing corresponding $3 \mathrm{D}$ 


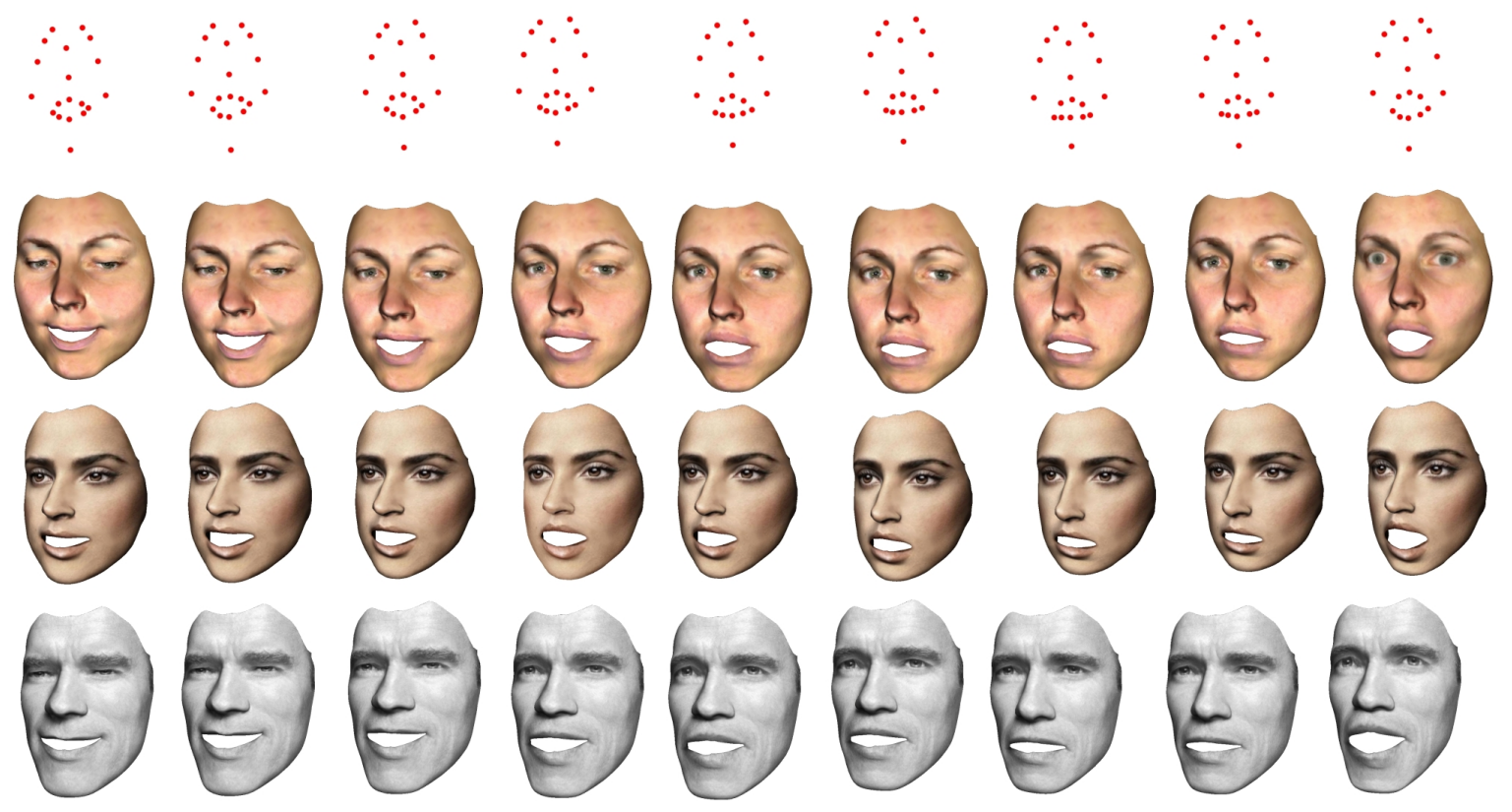

Figure 10: Selected frames from the animation results. Upper raw: original motion capture frames of expressions of the first subject, results of the mapping to the morphable model space for the same subject. Lower two raws: expression crossmapping to animate a single $3 D$ scan of another subject.
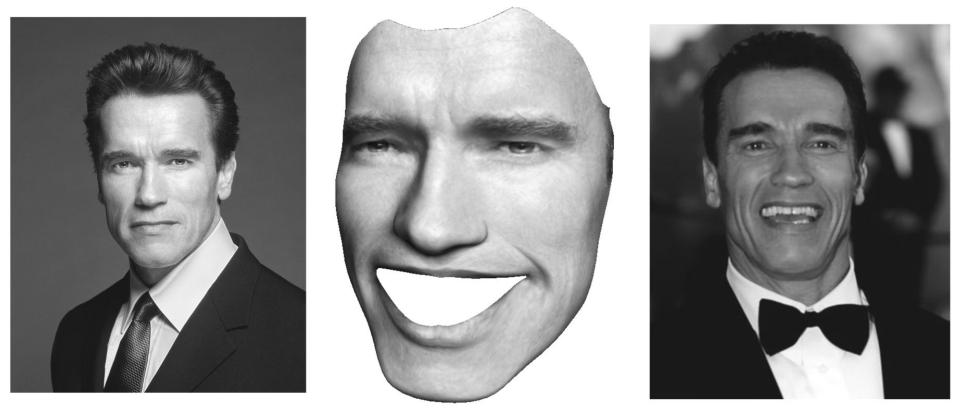

Figure 11: The comparison of the result of expression retargeting of happy expression. From left to right: expression transfer result for happy expression, original photograph; result of adding expression modified $2 D$ face into original photograph; a real photograph of the same subject with similar expression.

frames from their parameter vectors within the model space.

\section{Results and Evaluation}

\subsection{High-resolution animation from motion capture}

\subsubsection{Animation}

One of the possible evaluation scheme of produced facial animation is a frame-by-frame comparison of animation results to original motion capture data. Figure 10 presents such comparison. The first two rows of figure 10 the reader can see the animation results for a cross-mapping of facial dynamics from motion capture data to the dense 3D scans of the same person. As the reader may notice the dense 3D animation frames reflect the expressions changes which are present in the motion capture frames. A quantitative analysis of animation results is done here by comparing original motion capture points which are representative of facial expressions to corresponding vertices of the resulting 3D dense scan animation frames which were sampled at the locations where motion capture markers have been placed. The Y coordinate of the points on the middle of outer contour of upper and lower lips is displayed for the original motion capture data, our results are compared with key-frame interpolation (where the key-frames were selected by hand). Figures 12 (a) and (b) show that the results of our method give a smoother trajectory which is closer to original motion data than a standard key-frame interpolation approach. However, the results of our method are still far from a real motion capture coordinate positions. In future we plan to solve this by adding additional 
constraints during the registration step and improving the mapping procedure.

\subsection{Retargeting animation to a 3D model}

A comparison of a retargeted expression with an original photograph can be seen in figure 11. It shows an original photograph that is used here to estimate $3 \mathrm{D}$ shape and texture of the subject $B$, expression retargeting result for a happy expression and a real photograph of the same individual in the different lighting conditions performing a similar expression. One may see that although the results of expression retargeting preserve the realism of resulting expression, there are noticeable differences in the expression style from the real smile of the same individual. It happens due to the fact that proposed morphable model is based on the data of a single performer (performer $A$ ) and thus it can't accurately represent inter-personal variations for different expressions. Therefore, during the expression transfer stage it can't reliably predict specific personal characteristics of an individuals smile style.

The selected frames from the animation sequence of expression retargeting to animate the dense scans of another subjects are presented in the lower two rows of the figure 10. The results demonstrate that the current method works for the expression retargeting to animate a single photograph or 3D scan of other subjects. This is important as it allows automating of a standard animation pipeline when motion capture is used to animate a photograph or a 3D mesh of an performer which is not available for recording in the studio. The realism of expression retargeting can be increased in future by adding a personspecific model of facial expressions.

\section{Conclusion}

This paper has introduced a framework for high-resolution facial animation from sparse marker-based motion capture. The approach learns corresponding high and low resolution facial expression spaces from a set of 3D scans of a specific person. Radial basis function interpolation is used to map motion capture trajectories of facial dynamics from the low to high resolution spaces. Results demonstrate that this enables high-resolution animation of facial expressions with the detailed skin wrinkling and appearance captured in $3 \mathrm{D}$ face scans and the natural dynamics from motion capture. The approach allows automatic performance-based animation of a subject from motion capture sequences without the requirement for highly skilled and time-consuming manipulation of a facial model. Retargeting of the learnt facial model enables animation of a novel subject from a single photograph or 3D scan. The approach uses the difference in facial anatomy to retarget the motion capture to a new person. This allows the novel subject to be animated with the high-resolution details of skin wrinkling derived from the learnt person specific expression model. Results have been demonstrated for animation of models for celebrities derived from a single photograph. Currently this work is limited to facial expressions animation. Future research should address the problem of high-resolution animation of speech and emotion with the natural dynamics of real faces.

\section{Acknowledgements}

This work is supported by EPSRC Grant EP/C53879X Dynamic Faces: Understanding the Dynamics of Real Faces.

\section{References}

[1] V. Blanz and T. Vetter. A morphable model for the synthesis of 3d faces. In SIGGRAPH'99, volume 4, pages 187-194, Los Angeles, 1999. ACM Press.

[2] V. Blanz and T. Vetter. Face recognition based on fitting a 3D morphable model. IEEE Transactions on Pattern Analysis and Machine Intelligence, 25(9):10631074, September 2003.

[3] F. L. Bookstein. Principal warps: Thin-plate splines and the decomposition of deformations. IEEE Trans. Pattern Anal. Mach. Intell., 11(6):567-585, 1989.

[4] Z. Deng and U. Neumann. efase: expressive facial animation synthesis and editing with phoneme-isomap controls. In SCA '06: Proceedings of the 2006 ACM SIGGRAPH/Eurographics symposium on Computer animation, pages 251-260, Aire-la-Ville, Switzerland, Switzerland, 2006. Eurographics Association.

[5] R. M. Koch, M. H. Gross, F. R. Carls, D. F. von Büren, George Fankhauser, and Yoav I. H. Parish. Simulating facial surgery using finite element models. In SIGGRAPH '96: Proceedings of the 23rd annual conference on Computer graphics and interactive techniques, pages 421-428, New York, NY, USA, 1996. ACM Press.

[6] Y. Lee, D. Terzopoulos, and K. Walters. Realistic modeling for facial animation. In SIGGRAPH '95, pages 55-62, New York, NY, USA, 1995. ACM Press.

[7] P. Muller, G. A. Kalberer, M. Proesmans, and L.J. Van Gool. Realistic speech animation based on observed 3d face dynamics. 152(4):491-500, August 2005.

[8] J. Noh, D. Fidaleo, and U. Neumann. Animated deformations with radial basis functions. In VRST '00: Proceedings of the ACM symposium on Virtual reality software and technology, pages 166-174, New York, NY, USA, 2000. ACM Press.

[9] J. Noh and U. Neumann. Expression cloning. In Proc. Vision, Video and Graphics, VVG 2003, pages 277-288, New York, NY, USA, 2003. ACM Press.

[10] F. I. Parke. Computer generated animation of faces. In ACM'72: Proceedings of the ACM annual conference, pages 451-457, New York, NY, USA, 1972. ACM Press.

[11] F. Pighin, J. Hecker, D. Lischinski, R. Szeliski, and D. H. Salesin. Synthesizing realistic facial expressions from 


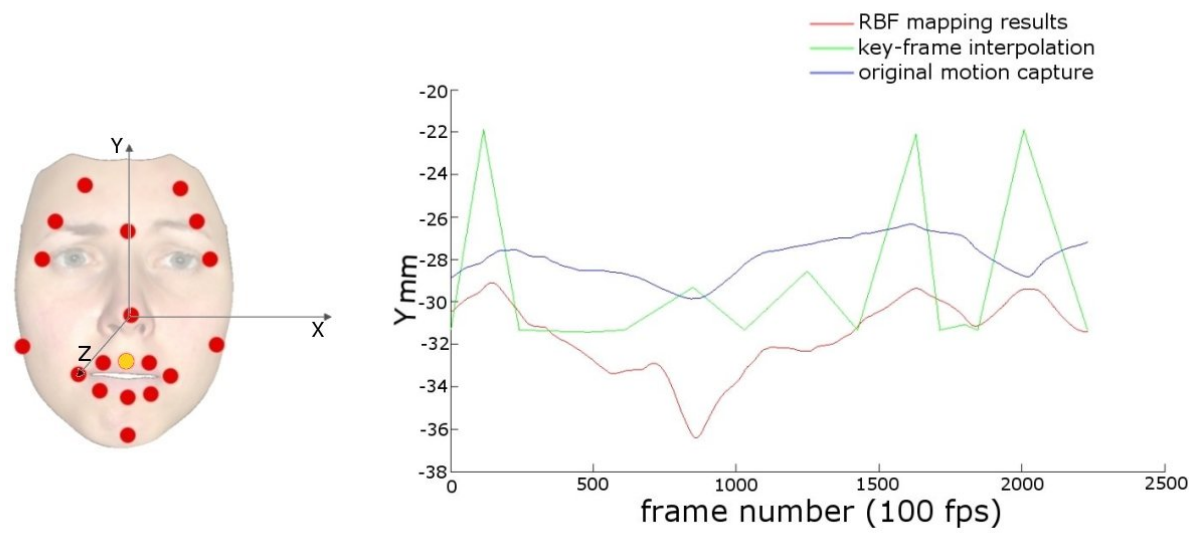

(a)

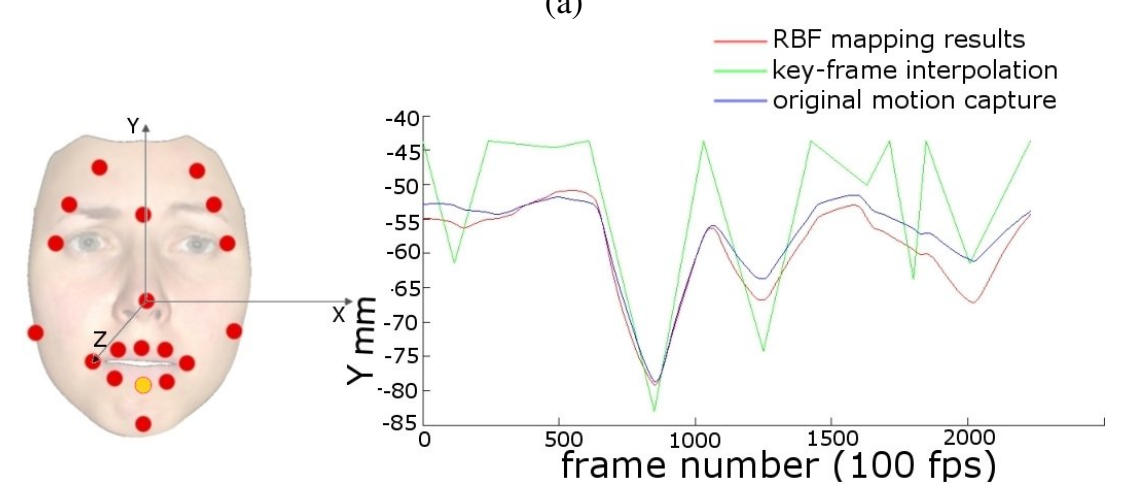

(b)

Figure 12: (a) Y coordinate of 3D point which corresponds to motion capture sensor location on the middle of outer contour of the upper lip plotted for consecutive frames.

(b) Y coordinate of 3D point which corresponds to motion capture sensor location on the middle of outer contour of the lower lip plotted for consecutive frames.

photographs. In SIGGRAPH '98: Proceedings of the 25th annual conference on Computer graphics and interactive techniques, pages 75-84, New York, NY, USA, 1998. ACM Press.

[12] D. Stork R. Duda, P. Hart. Pattern Classification. John Wiley and Sons, New York, USA, 2001.

[13] M. L. Sanchez, J. D. Edge, S. A. King, and $\mathrm{S}$. Maddock. Use and re-use of facial motion capture data. In SIGGRAPH '01: Proceedings of the 28th annual conference on Computer graphics and interactive techniques, pages 135-142, University of Bath, UK, 2003.

[14] K. Singh and E. Fiume. Wires: a geometric deformation technique. In SIGGRAPH '98: Proceedings of the 25th annual conference on Computer graphics and interactive techniques, pages 405-414, New York, NY, USA, 1998. ACM Press.
[15] K. Singh and E. Kokkevis. Skinning characters using surface oriented free-form deformations. In Graphics Interface, pages 35-42, 2000.

[16] J. R. Tena, M. Hamouz, A.Hilton, and J. Illingworth. A validated method for dense non-rigid 3D face registration. In Proc. of International Conference on Video and Signal Based Surveillance (AVSS 06), pages 81-81, November 2006.

[17] J. R. Tena, M. Hamouz, A. Hilton, and J. Illingworth. A validated method for dense non-rigid $3 \mathrm{~d}$ face registration. In AVSS '06, page 81, Washington, DC, USA, 2006. IEEE Computer Society.

[18] L. Williams. Performance-driven facial animation. In SIGGRAPH '90: Proceedings of the 17th annual conference on Computer graphics and interactive techniques, pages 235-242, New York, NY, USA, 1990. ACM Press. 